\title{
Granulocyte Colony-Stimulating Factor Treatment Prevents Cognitive Impairment Following Status Epilepticus in Rats
}

\author{
Yong Zhang, ${ }^{a, b}$ WenYong Wang,${ }^{c}$ ZhiJian Sun,,${ }^{d}$ DongYun Feng,${ }^{a}$ YanChun Deng,,${ }^{a}$ YongHong Liu, ${ }^{a}$ \\ Gang ZhaO, ${ }^{a}$ HuaNing WANG, ${ }^{e}$ and YuanGui HuAnG ${ }^{*}, a$ \\ ${ }^{a}$ Department of Neurology, Xijing Hospital, The Fourth Military Medical University; ${ }^{c}$ Department of Pathology, The \\ Fourth Military Medical University; ${ }^{d}$ Department of Histology and Embryology, The Fourth Military Medical University; \\ ${ }^{e}$ Department of Psychiatry, Xijing Hospital, The Fourth Military Medical University; Xi'an 710032, China: and \\ ${ }^{b}$ Department of Neurology, The PLA 187th Hospital; Haikou 571159, China. \\ Received November 23, 2009; accepted January 19, 2010; published online January 26, 2010
}

Status epilepticus (SE) rendered selective neuronal loss and cognitive impairments. Previous studies proved that granulocyte colony-stimulating factor (G-CSF) acted as a neuroprotectant in some nervous diseases. However, no investigations were focused on whether G-CSF could protect the hippocampus from SE. In this study, we administered recombinant human G-CSF into Sprague-Dawley rats with lithium-pilocarpine-induced SE subcutaneously for three times. The Morris water maze was employed to determine spatial learning ability from the 15th to 20th days after the treatment. The quantitative terminal deoxynucleotidyl transferase-mediated deoxyuridine triphosphate (dUTP) nick end labeling (TUNEL) staining and levels of apoptosis-related molecules including cleaved caspase-3, Bcl-xL and Bax on hippocampal CA1 region were examined by immunohistochemical staining at the 3rd and the 5th day after the treatment. Moreover, the phosphorylation of AKT was evaluated with Western blot at the 6th, 24th and 48th hours after the treatment to explore apoptosis and detect the protective effects of G-CSF. We found G-CSF treatment prevented SE-induced cognitive impairments with the decreased escape latency time on the 17 th $(29.86 \pm 9.09$ vs. $38.33 \pm 6.94, p<0.05)$ and 18 th days $(23.83 \pm 6.17 v s$. $33.52 \pm 8.48, p<0.05)$. The reduced TUNEL staining demonstrated reduced neuronal apoptosis occurrences. The anti-apoptotic effects were associated with decreased cleaved caspase-3 and Bax expression and increased phosphorylation of AKT and Bcl-xL expression. Taken together, our results suggested that systemic G-CSF treatment conducted neuroprotective function following $\mathrm{SE}$ through an anti-apoptotic pathway and prevented cognitive impairments, which may provide novel insights into pathogenesis and treatment following SE injury.

Key words granulocyte colony-stimulating factor; status epilepticus; cognitive impairment

Epilepsy is a common, chronic neurological disorder affecting up to $3 \%$ of the population of all ages, and is characterized by recurrent, unprovoked seizures. ${ }^{1)}$ Status epilepticus (SE) is a state of continuous seizures lasting more than 5 min, or rapidly recurrent seizures without regaining consciousness. ${ }^{2)}$ SE can increase the risk of subsequent seizures and cause profound cerebral damages, accompanied by a characteristic pattern of neuronal cell loss that occurs preferentially in the hippocampus. Studies on the clinical and experimental material revealed that seizure-induced neuronal death including necrotic and apoptotic forms within the vulnerable areas. ${ }^{3,4)}$ Such injury may arouse impairments on cognitive functions, epilepsy or even death. ${ }^{5)}$

In spite of the introduction of new pharmacological therapies and the increased success rate of surgical treatments for epilepsy, a substantial number of patients either did not get seizure-free or they endured major adverse events. ${ }^{6)}$ Therefore, it is an urgent task to explore the exact mechanism of apoptosis induced by seizures and find effective therapeutic reagents. So far, several adjuvant biologics such as erythropoietin were attempted to be applied in the rat epilepsy models and the reduced SE-related neuron death was observed. ${ }^{7,8)}$

In recent years, accumulating evidences have proved that granulocyte colony-stimulating factor (G-CSF), an endogenous hematopoietic factor, mediates neuroprotection in experimental models of focal and global cerebral ischemia, Parkinson's disease, spinal cord injury and allergic encephalomyelitis. ${ }^{9-13)}$ Both G-CSF and G-CSF receptor (GCSFR) distributed widely on the central nervous system
(CNS) neurons. ${ }^{14)}$ They were up-regulated under certain pathological conditions through an autocrine protective signaling mechanism. Specific G-CSFR expression on hippocampal CA1 region was also identified. ${ }^{15)}$ Additionally, exogenous G-CSF could penetrate the blood-brain barrier via receptor-involved mechanism. ${ }^{16)}$ G-CSF mediated neuroprotection in experimental studies of cerebral ischemia through different mechanisms including mobilization of hematopoietic stem cells, anti-apoptosis, increase of neuronal differentiation, angiogenesis and anti-inflammation. ${ }^{17-19)}$ Moreover, G-CSF attenuated glutamate-induced cell death of cerebellar granule neurons in vitro. ${ }^{20)}$ During the pathogenesis of SE-induced neuronal apoptosis, hypoxia/ischemia and synaptically released glutamate acting on ionotropic and metabotropic receptors were involved. ${ }^{21)}$ Although no direct evidences showed whether G-CSF was applicable during SE, the previous studies on G-CSF functions made us hypothesize that exogenous G-CSF would be of value in reducing neuronal injury following SE. In this study, we examined the effect of G-CSF treatment on neuronal death in hippocampus and cognitive impairment during lithium-pilocarpine induced SE. The sacrificed rats were evaluated by analysis on several apoptosis-related markers including terminal deoxynucleotidyl transferase-mediated deoxyuridine triphosphate (dUTP) nick end labeling (TUNEL), cleaved caspase-3, BclxL, Bax, phosphorylated AKT and water maze. Finally, the results showed that G-CSF conducted neuroprotective function during SE through an anti-apoptotic pathway and prevented the resulting cognitive impairment. 


\section{MATERIALS AND METHODS}

Animals and Lithium-Pilocarpine Induced Model of Epilepsy Adult male Sprague-Dawley rats weighing 180 $200 \mathrm{~g}$ were purchased from the Animal Center of the Fourth Military Medical University and their care and handling was conducted in compliance with the Chinese Animal Welfare Act and approved by the responsible government agency at the Fourth Military Medical University.

A total of 160 rats were randomly assigned to four groups and used to test several indicators at the scheduled time: the control rats treated with saline, rats administered with G-CSF alone (G-CSF), rats administered with lithium-pilocarpine (L-P) and rats administered with both G-CSF and lithium-pilocarpine $(\mathrm{G}+\mathrm{L}-\mathrm{P})$. In the $\mathrm{L}-\mathrm{P}$ and $\mathrm{G}+\mathrm{L}-\mathrm{P}$ groups, all rats received lithium chloride $(3 \mathrm{mEp} / \mathrm{kg}$, intraperitoneally (i.p.); Sigma-Aldrich, St. Louis, MO, U.S.A.) at $24 \mathrm{~h}$ prior to the administration of pilocarpine. Then, rats were pretreated with methylscopolamine $(1 \mathrm{mg} / \mathrm{kg}$, i.p.; Sigma-Aldrich, St. Louis, MO, U.S.A.) at $15 \mathrm{~min}$ prior to pilocarpine treatment to reduce the peripheral effects of the convulsant. One single dose of pilocarpine hydrochloride $(30 \mathrm{mg} / \mathrm{kg}$, i.p., Sigma) was administered. Seizures were scored in each rat by Racine's scale, $^{22)}$ and the durations of SE were determined by behavioural assessment, considering only convulsive (motor) seizures for scoring: stage 4 , rearing; stage 5 , rearing plus loss of balance and falling, accompanied by generalized clonic seizures. Diazepam $(10 \mathrm{mg} / \mathrm{kg}$, i.p., Xijing Hospital Pharma, Xi'an, China) was administered $60 \mathrm{~min}$ after the onset of SE to terminate convulsion in all rats. The beginning of SE was defined as the onset of continuous generalized (stage 4 or 5 by Racine's scale) seizure activity without regaining normal behavior between the seizures.

G-CSF Treatment In the G-CSF and G+L-P groups, recombinant human granulocyte-colony stimulating factor (G-CSF, $50 \mu \mathrm{g} / \mathrm{kg}$, Neupogen (R), Amgen, Thousand Oaks, CA, U.S.A.) was given to the rats subcutaneously for three times: $12 \mathrm{~h}$ before pilocarpine treatment, right after diazepam treatment and $24 \mathrm{~h}$ after diazepam treatment. The three-dose sequence was chosen to maximize the potential protective effect of the drug and to mimic a treatment regimen that could theoretically be useful as a preoperative therapy in humans. Normal saline injections in similar volumes (approximately $0.05 \mathrm{ml}$ per injection) were given to the untreated groups on the same dosing schedule as G-CSF treatment.

Water Maze Cognitive function was tested by the water maze ${ }^{23)}$ which was a circular tank with a diameter of $1.8 \mathrm{~m}$. The tank was filled with opaque water $(35 \mathrm{~cm}$ depth, $21 \pm 1^{\circ} \mathrm{C}$ ) made by the addition of a non-toxic paint. A $10 \mathrm{~cm}$-diametered platform submerged $2 \mathrm{~cm}$ below the surface of the water was placed in the center of one quadrant of the pool. Four points, equally spaced along the circumference of the pool, were arbitrarily assigned as: $\mathrm{N}, \mathrm{E}, \mathrm{S}$ and $\mathrm{W}$, on this basis, the pool area was divided into 4 quadrants (NE, SE, SW and NW). These points served as the starting positions at which the rat was lowered gently into the water. The location of the escape platform was fixed (in the middle of quadrant NE) throughout training. Latencies to find the platform, distance swum, time and distance in each quadrant were automatically recorded using a Videomex-V Image Motion System (provided by the Chinese Academy of Medical
Sciences). Each trial was started and ended manually by the experimenter, who operated a remote switch connected to the microcomputer. On the day before the trials, each rat was placed in the pool for $60 \mathrm{~s}$ without the platform present for habituating the training environment. Rats were submitted to daily sessions of four trials per day for $5 \mathrm{~d}$ for acquisition phase assays from the 15 th days on. At the beginning of each trial, the rat was gently placed into the water at the start location with its head facing the wall of the water maze in order to negate any directional influence at the onset of the trial. The starting location was varied in each trial, and the sequence of start locations was randomized across days. A trial ended when the rat escaped onto the platform. If a rat failed to escape within $60 \mathrm{~s}$, it was guided to the platform by the experimenter. The rat was allowed to spend $10 \mathrm{~s}$ on the escape platform and then placed for $20 \mathrm{~s}$ in a holding cage before the next trial began, resulting in an inter-trial interval of about $35 \mathrm{~s}$. At the end of the session, the rat was dried with a towel before being returned to its home cage. On each trial, the time needed to reach the platform was measured. The mean of the escape latencies of every daily trials was elaborated statistically.

One day after the last training session, each rat was subjected to a probe trial $(60 \mathrm{~s})$ in which the platform was removed from the pool. The rats were placed in the water maze in the south position (S) because in the acquisition phase, the platform was in the northeast position. The time spent in the quadrant of the former platform position and the correct annulus crossing, i.e., the number of times animals passed through the circular area $(10 \mathrm{~cm})$ that formerly contained the submerged platform during acquisition, were taken as measures for spatial memory.

Immunohistochemistry and TUNEL Rats were euthanized under general anesthesia (thionembutal, $50 \mathrm{mg} / \mathrm{kg}$, i.p.) and perfused with phosphate buffered saline (PBS) (100 ml) and $4 \%$ paraformaldehyde sequentially by trans-cardiac approach for histology. Three-micrometer thick sections were mounted on poly-L-lysine-coated slides, followed by deparaffinization in xylene and dehydration in graded alcohol. The endogenous peroxidase activity was blocked by an incubation with $3 \mathrm{ml} / \mathrm{ml} \mathrm{H}_{2} \mathrm{O}_{2}$ in methanol for $30 \mathrm{~min}$. Tissue sections were subjected to antigen retrieval by boiling in $0.01 \mathrm{~mol} / 1$ sodium-citrate $(\mathrm{pH} 6)$ for $10 \mathrm{~min}$ in a microwave oven. After blocked with $15 \mathrm{mg} / \mathrm{ml}$ normal goat serum for $15 \mathrm{~min}$, immunohistochemistry assay was carried out with an Elivision plus staining kit (Maixub-bio) according to its mannual. In brief, tissue sections were stained with rabbit anti-cleaved caspase 3 (1:100, Cell Signaling Technology, Boston, MA, U.S.A., \#9661), mouse anti-Bcl-xL (1:50, Santa Cruz Biotech, Santa Cruz, CA, U.S.A., SC-8392) or anti-Bax ( $1: 50$, SC-7480) overnight at $4{ }^{\circ} \mathrm{C}$ and washed three times, after which an enhancer was added for $30 \mathrm{~min}$, followed by three washes before appropriate horse-radish peroxidase (HRP)-conjugated secondary antibodies (DAKO, Glostrup, Denmark) were added and washed another three times. Finally, diaminobenzidine (DAB) was added for coloring and observed under a microscope.

The TUNEL staining was done with a kit (Roche, Indianapolis, IN, U.S.A., \#11684795910) according to the manufacturer's protocol. In brief, the treated tissue slides were rinsed twice with PBS for 5 min and incubated for $1 \mathrm{~h}$ in a 
humidified container at $37^{\circ} \mathrm{C}$ with a TUNEL reaction mixture containing the following: $5 \mu \mathrm{l}$ enzyme solution (terminal deoxynucleotidyl transferase in storage buffer); $50 \mu$ l label solution (fluorescein-nucleotide mixture in reaction buffer). After washing three times in PBS, slides were directly observed under a fluorescence microscope.

Quantitative Cell Count Brains located between 3.3 and $4.5 \mathrm{~mm}$ posterior to bregma (encompassing the dorsl hippocampal region) were sectioned in a serial manner, and a total of approximately 30 slices per animal were saved, taking care to avoid adjacent slices. To reduce counting bias, only central cell profiles that exceeded $3 \mathrm{~mm}$ were included. As there was no obvious difference in cell profiles between hemispheres, values for right and left hemispheres were averaged in each rat. The numbers of labeled cells in three coronal sections were counted using $400 \times$ magnifications and by eye on a blind rating scale independently by two people, and then expressed as average number of cells within per section ( 6 sections per animal, $n=5$ for each group).

Western Blot Activation of AKT was assayed by Western blott following standard protocols. ${ }^{24)}$ After sacrifice, brains were removed and frozen in liquid nitrogen. The frozen tissue blocks were homogenized in lysis buffer $(50$ $\mathrm{mmol} / \mathrm{l}$ Tris-Cl, $2.5 \mathrm{mmol} / \mathrm{l}$ ethylene glycol bis(2-aminoethyl ether)- $N, N, N^{\prime}-N^{\prime}$-tetraacetic acid (EGTA), $1 \mathrm{mmol} / 1$ ethylenediaminetetraacetic acid (EDTA), $10 \mathrm{mmol} / 1 \mathrm{NaF}, 1 \%$ deoxycorticosterone, $1 \%$ Triton $\mathrm{X}-100,1 \mathrm{mmol} / 1$ phenylmethylsulfonyl fluoride, and $2 \mathrm{mmol} / 1 \mathrm{Na}_{3} \mathrm{VO}_{4}$ ). The protein concentration in the supernatant was measured using the BCA protein assay Kit (Pierce, Rockford, IL, U.S.A.). Proteins $(25 \mu \mathrm{g})$ from whole cell lysates were separated by $12 \%$ sodium dodecyl sulfate-polyacrylamide gel electrophoresis (SDS-PAGE) and transferred onto nitrocellulose membrane. The blots were blocked for $2 \mathrm{~h}$ at room temperature with $5 \%$ nonfat dry milk in Tris-buffered saline (TBS), then incubated with the primary antibodies: rabbit anti-phosphorylated-AKT 1/2/3 (1:200, Santa Cruz Biotech, SC-16646) or goat antibeta actin $\left(1: 200\right.$, Santa Cruz Biotech, SC-1615) at $4{ }^{\circ} \mathrm{C}$ overnight. After three washing steps with Tris-buffered salt solution (TTBS), the membranes were incubated with horseradish peroxidase-conjugated donkey anti-rabbit or anti-rabbit antibodies ( $1: 2000$, Sigma) for $1 \mathrm{~h}$ at room temperature. Proteins recognized by antibody labeling were detected using enhanced chemiluminescence according to the manufacturer's instructions. Pre-stained protein markers were used for molecular weight determinations. Bands were quantified by optical density method (Image J), and the densities were expressed relative to beta actin.

Statistics A chi-squared test was used for a comparison of mortality. The data of Morris water maze task was compared using three-way mixed analysis of variance (ANOVA) $(\mathrm{L}-\mathrm{P} \times \mathrm{G}-\mathrm{CSF} \times$ Day). To compare the latencies to the platform and the time spent in the target quadrant, values among Western blot and cell counts, one-way analysis of variance (ANOVA) was applied. All values are mean \pm standard error of mean (S.E.M.), except where otherwise indicated. Statistical significance was accepted at $p<0.05$.

\section{RESULTS}

G-CSF Attenuated Body Weight Loss Induced by
Lithium-Pilocarpine Treatment Body weight is a classical parameter of development. To assess the influence of SE on the tested rats, we divided 60 rats into four groups $(n=15$ for each) and weighed them daily after the treatment. No obvious body weight changes were found among the control and G-CSF-treated groups. The daily body weight of the $\mathrm{G}+\mathrm{L}-\mathrm{P}$ group had an initial trend of being improved compared with the L-P group (Fig. 1). On the third $(0.89 \pm 0.02 \%$ vs. $0.72 \pm 0.15 \%)$ and fourth day $(0.98 \pm 0.03 \% \quad v s$. $0.82 \pm 0.04 \%)$, obvious differences $(p<0.05)$ were present between the G+L-P and L-P groups. Finally, four groups had equivalent body weights by the fourteenth day.

G-CSF Treatment Prevented Cognitive Impairment Following SE in Rats The Morris water maze test was conducted to assess spatial learning and memory controlled by hippocampus. To judge whether G-CSF treatment prevented cognitive impairment, the rats evaluated by body weights changes were subjected to water maze test from the fifteenth day after the treatment. All rats swam at the similar speed without impairment of mobility $(p>0.1)$. All groups had tendency of reduced latency to find the hidden platform as training progressed $\left(F_{3,60}=35.21, p<0.01\right)$. There was no significant difference between the control and G-CSF treated groups within each testing day. Although the rats treated with G+L-P or L-P spent more time than the control and G-CSF treated groups, no significant difference between them was found before the third tested day $(p>0.05)$. The rats treated with L-P or G+L-P showed impaired performance compared with the other two groups on the third and the fourth day for the significant difference was observed between these groups. EL time spent by L-P and G+L-P was significantly different $(p<0.05)$ on the third $(38.33 \pm 6.94 \mathrm{~s} v s .29 .86 \pm$ $9.09 \mathrm{~s})$ and the fourth $(33.52 \pm 8.48 \mathrm{~s} v s .23 .83 \pm 6.17 \mathrm{~s})$ day. On the fifth day, rats of L-P group spent significantly more time than the control and G-CSF treated groups. However, no obvious difference $(p>0.05)$ was found between the L-P $(20.29 \pm 6.19 \mathrm{~s})$ and $\mathrm{G}+\mathrm{L}-\mathrm{P}(13.66 \pm 7.76 \mathrm{~s})$ groups. The data were presented in Fig. 2A.

Figure 2B presents the percentage of time spent in the target quadrant where the platform had been previously placed on the 20th day after the treatment. ANOVA results showed that rats treated with L-P spent less time in the target quadrant than the control group $(25.39 \pm 5.23 \%$ vs. $36.45 \pm 5.1 \%$ $p<0.05)$. No differences found either between the G-CSF, $\mathrm{G}+\mathrm{L}-\mathrm{P}$ and the control groups $(36.45 \pm 5.1 \%, 34.73 \pm$

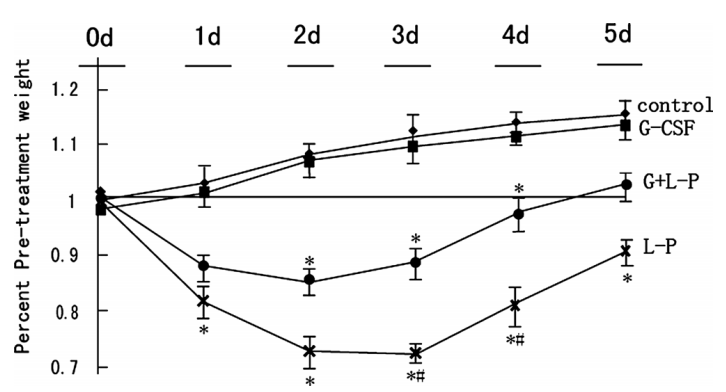

Fig. 1. Daily Body Weight of Tested Rats within $5 \mathrm{~d}$ after Treatment ( $n=15$ in Each Group)

The values were expressed as percent of pre-treatment body weight of the control group. $* p<0.05$, significant difference to the control group; $\# p<0.05$, significant difference to the G+L-P treated group. 
$11.81 \%$ vs. $37.53 .45 \pm 4.7 \%, p>0.05)$ or between the L-P and $\mathrm{G}+\mathrm{L}-\mathrm{P}$ groups $(p>0.05)$.

G-CSF Reduced Neuronal Apoptosis on Hippocampal CA1 Region The results about water maze informed us

A

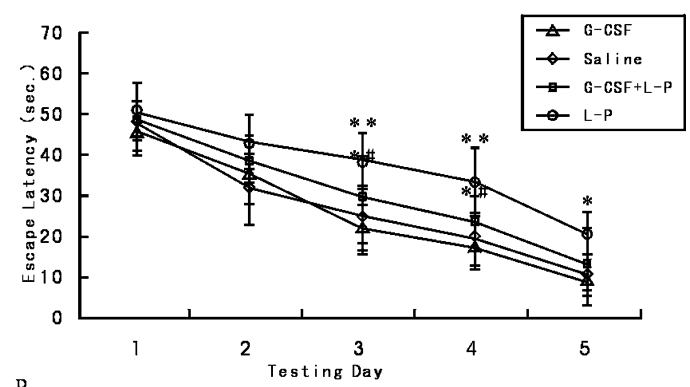

B

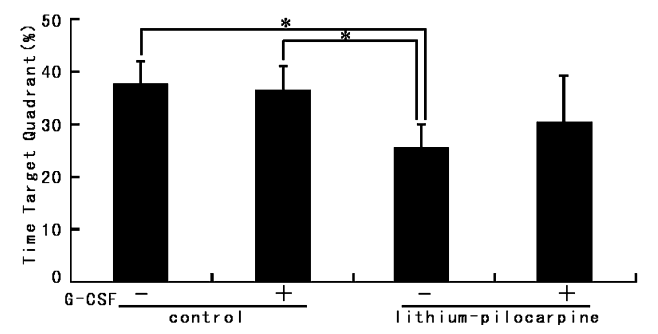

Fig. 2. Comparisons of the Performance of the Morris Water Maze Task in the Control, G-CSF, Lithium-Pilocarpine, and G-CSF Plus LithiumPilocarpine Treated Groups ( $n=15$ in Each Group)

(A) The mean escape latencies (s) to the hidden platform in water maze as a function of day. (B) Probe trials. The percentages of time spent in the target quadrant where the platform was located during acquisition test. $* * p<0.01, * P<0.05$, significant difference to the control group. ${ }^{\#} p<0.05$, significant difference to the L-P treated group.
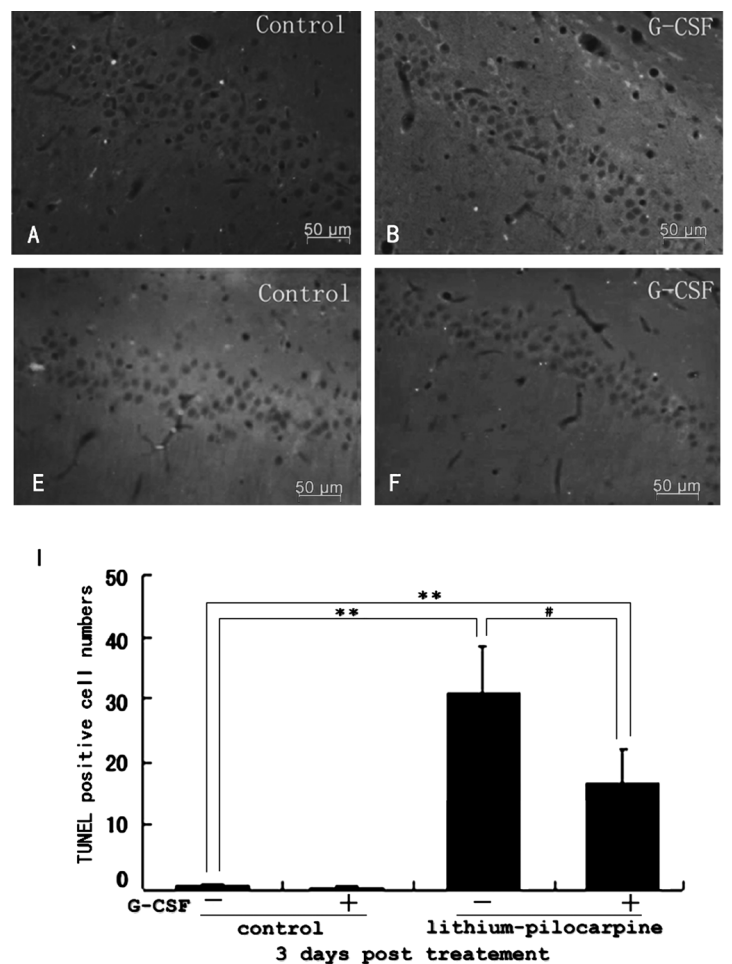

that G-CSF may exert potential protection on hippocampus. To identify the exact mechanism, TUNEL staining, a common method for detecting DNA fragmentation that results from apoptotic signaling cascades, was applied. On the third and fifth days after the treatment, few TUNEL-positive cells were observed in the control and G-CSF groups (Figs. 3A, $3 \mathrm{~B}, 3 \mathrm{E} 3 \mathrm{~F}, 2 \pm 1.3,1.8 \pm 1.5,1.8 \pm 0.9$ and $1.5 \pm 1.2$, respectively). In contrast, TUNEL-positive cells were abundant in the L-P group and increased sharply from the third day $(30.8 \pm 7.8, p<0.01$, Fig. $3 \mathrm{C})$ to the fifth day $(50.7 \pm 18.6$, $p<0.01$, Fig. $3 \mathrm{G}$ ), representing significant difference compared with the control group (Figs. 3I, 3J). TUNEL-positive cells were also found both on the third (Fig. 3D, 16.7 \pm 5.4 ) and the fifth day (Fig. $3 \mathrm{H}, 30.2 \pm 7.8$ ) after the treatment, albeit sparsely, in the G+L-P group. However, when compared with the L-P group, G-CSF treatment decreased the total number of TUNEL-positive cells by $43 \%$ on the third day (Fig. 3I, $p<0.05$ ) and $40 \%$ on the fifth day (Fig. 3J, $p<0.01$ ), respectively.

Effect of G-CSF Treatment on Bax Expression In order to explore the anti-apoptotic and pro-apoptotic molecules adjusted by G-CSF treatment, we examined Bax, Bcl$\mathrm{xL}$ and cleaved caspase-3 on hippocampal CA1 area. Immunohistochemistry labeling demonstrated that the number of Bax positive cells increased significantly in the L-P and G+L-P groups relative to the control group both on the third (Figs. 4C, 4D, 23.6 $\pm 4.7,13.4 \pm 4.2$ vs. $3.5 \pm 2.3$ ) and fifth days after the treatment (Figs. $4 \mathrm{G}, 4 \mathrm{H}, 18.3 \pm 5.2,9.7 \pm 5.1$ vs. 2.9 \pm 1.8 ). Meanwhile, the number of Bax positive cells in the L-P and G+L-P groups declined on the fifth day. We also found that Bax expression in the L-P group was significantly
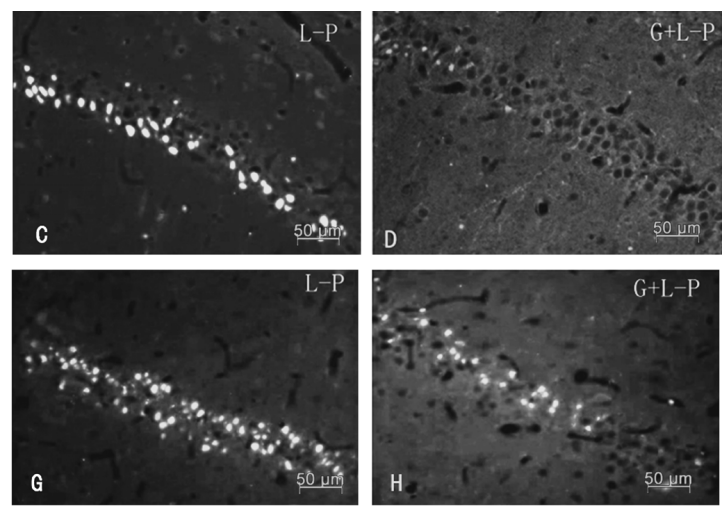

$J$

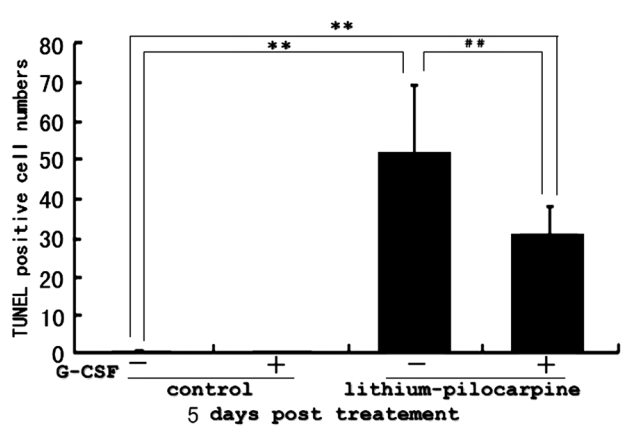

Fig. 3. TUNEL Assay on the Hippocampal CA-1 Region of Each Group on Days 3 and Days 5 after Treatment

(A) The control group on Days 3 ( $n=5$ ); (B) the G-CSF treated group on Days 3 ( $n=5$ ); (C) the lithium-pilocarpine treated group on Days 3 ( $n=5$ ); (D) the G-CSF plus lithiumpilocarpine treated group on Days $3(n=5)$; (E) the control group on Days $5(n=5)$; (F) the G-CSF treated group on Days $5(n=5)$; $(\mathrm{G})$ the lithium-pilocarpine treated group on Days $5(n=5)$; (H) the G-CSF plus lithium-pilocarpine treated group on Days $5(n=5)$; (I) TUNEL-positive neuron counts on Days 3; (J) TUNEL-positive neuron counts on Days 5. ** $p<0.01$, significant difference to the control group; $\# p<0.01$, ${ }^{\prime} p<0.05$, significant difference to the G+L-P treated group. 
different from the G+L-P group both on the third (Fig. 4I, $p<0.05$ ) and the fifth days (Fig. $4 \mathrm{~J}, p<0.05$ ). The obvious difference between distinct groups was illustrated on the Fig. 4.

Effect of G-CSF Treatment on Bcl-xL Expression The anti-apoptotic $\mathrm{Bcl}-\mathrm{xL}$ protein was expressed abundantly within the saline-treated control group (Figs. 5A, 5E) and GCSF induced its expression significantly both on the third (Figs. 5B, 5I, 58.6 20.2 vs. 22.3 $\pm 12.8, p<0.01$ ) and fifth days after the treatment (Figs. 5F, 5J, 66.6 $27.2 v s$.
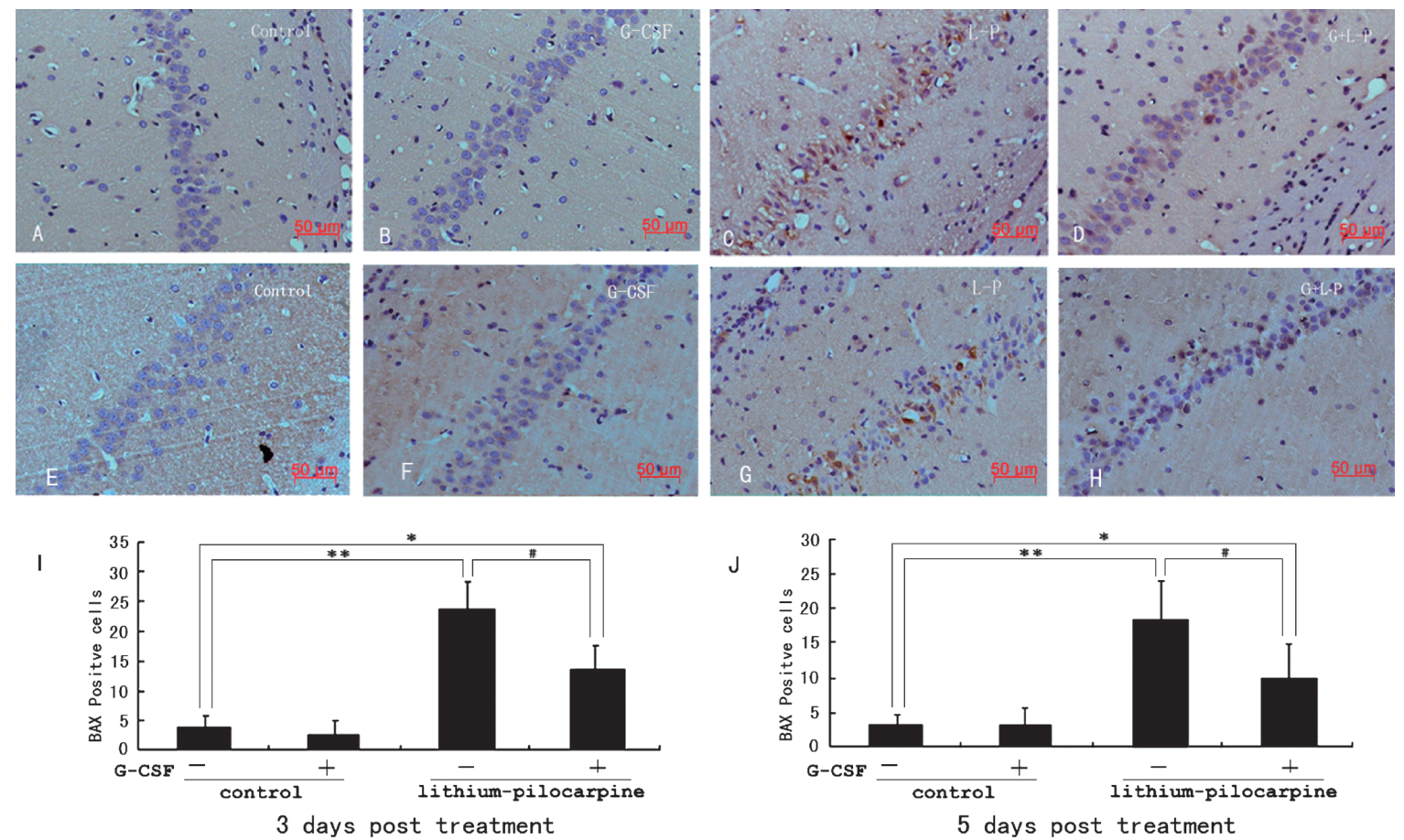

Fig. 4. Bax Staining on the Hippocampal CA-1 Region of Each Group on Days 3 and Days 5 after Treatment

(A) The control group on Days $3(n=5)$; (B) the G-CSF treated group on Days $3(n=5)$; (C) the lithium-pilocarpine treated group on Days 3 ( $n=5$ ); (D) the G-CSF plus lithiumpilocarpine treated group on Days $3(n=5)$; (E) the control group on Days $5(n=5)$; (F) the G-CSF treated group on Days $5(n=5)$; (G) the lithium-pilocarpine treated group on Days $5(n=5)$; $(\mathrm{H})$ the G-CSF plus lithium-pilocarpine treated group on Days $5(n=5)$; (I) Bax-positive neuron counts on Days 3 ; (J) Bax-positive neuron counts on Days 5 . $* * p<0.01, * p<0.05$, significant difference to the control group; ${ }^{*} p<0.05$, significant difference to the $\mathrm{G}+\mathrm{L}-\mathrm{P}$ treated group.
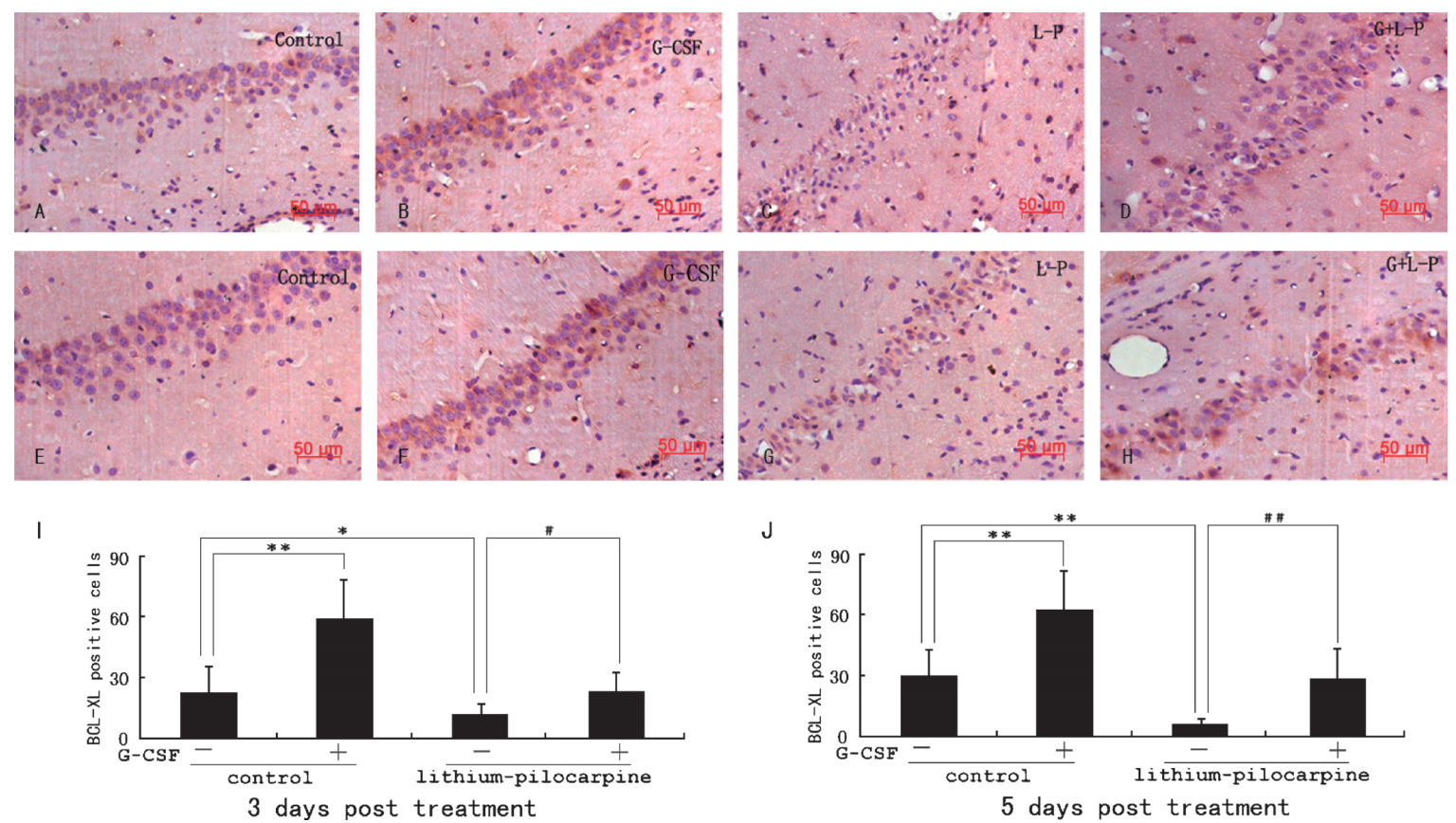

Fig. 5. Bcl-xL Staining on the Hippocampal CA-1 Region of Each Group on Days 3 and Days 5 after Treatment

(A) The control group on Days 3 ( $n=5$ ); (B) the G-CSF treated group on Days 3 ( $n=5$ ); (C) the lithium-pilocarpine treated group on Days 3 ( $n=5$ ); (D) the G-CSF plus lithiumpilocarpine treated group on Days $3(n=5)$; (E) the control group on Days $5(n=5)$; (F) the G-CSF treated group on Days $5(n=5)$; $(\mathrm{G})$ the lithium-pilocarpine treated group on Days $5(n=5)$; (H) the G-CSF plus lithium-pilocarpine treated group on Days 5 ( $n=5$ ); (I) Bcl-xL-positive neuron counts on Days 3; (J) Bcl-xL-positive neuron counts on Days 5. $* * p<0.01, * p<0.05$, significant difference to the control group; $\# p<0.01$, $\# p<0.05$, significant difference to the G+L-P treated group. 
29.4 $\pm 12.3, p<0.01)$. Bcl-xL expression was decreased markedly by L-P treatment compared with the control group both on the third (Figs. 5F, 5I, 11.8 \pm 9.2 vs. 22.3 \pm 12.8 , $p<0.05$ ) and the fifth days (Figs. 5G, 5J, 5.9 $\pm 2.1 v s$. $29.4 \pm 12.3, p<0.01)$. However, the supplemented G-CSF increased Bcl-xL expression to the level of the control group and showed significant difference to the L-P treated group both on the third (Figs. 5D, 5I, 21.3 \pm 9.9 vs. 11.8 \pm 9.2 , $p<0.05$ ) and the fifth days (Figs. 5H, 5J, 27.9 13.9 vs.
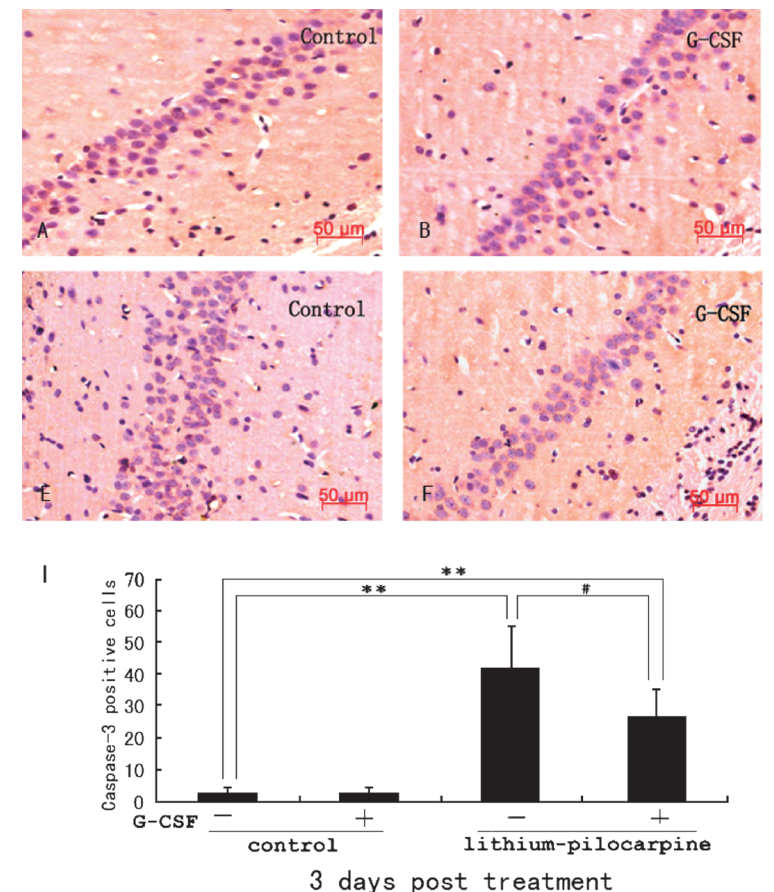
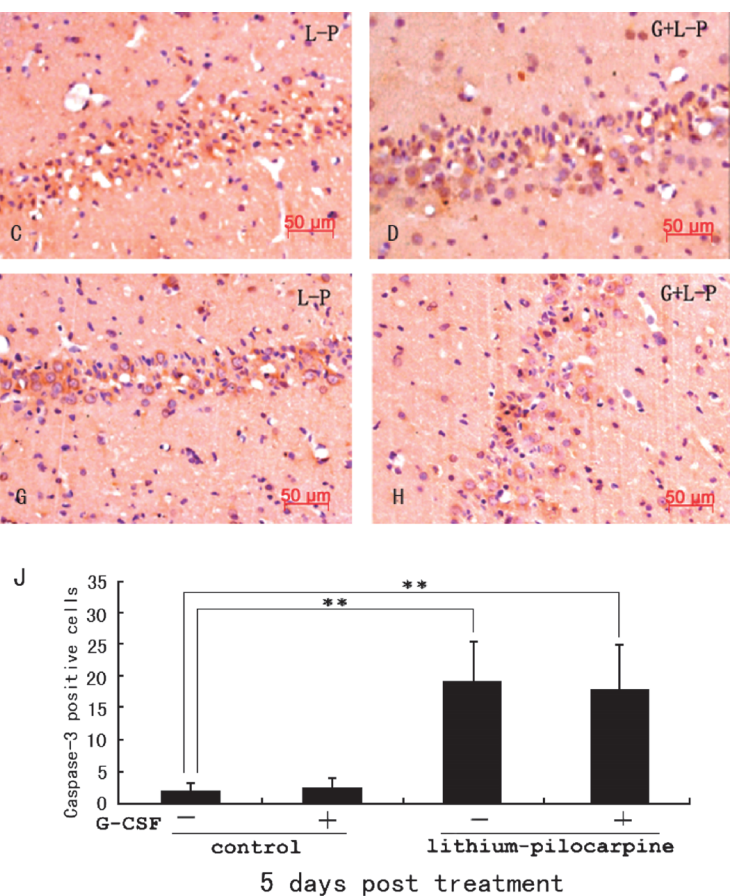

Fig. 6. The Cleaved Caspase-3 Staining on the Hippocampal CA-1 Region of Each Group on Days 3 and Days 5 after Treatment

(A) The control group on Days 3 ( $n=5$ ); (B) the G-CSF treated group on Days $3(n=5)$; (C) the lithium-pilocarpine treated group on Days 3 ( $n=5$ ); (D) the G-CSF plus lithiumpilocarpine treated group on Days $3(n=5)$; (E) the control group on Days $5(n=5)$; (F) the G-CSF treated group on Days $5(n=5)$; (G) the lithium-pilocarpine treated group on Days $5(n=5)$; (H) the G-CSF plus lithium-pilocarpine treated group on Days $5(n=5)$; (I) The cleaved caspase-3-positive neuron counts on Days 3; (J) The cleaved caspase-3-positive neuron counts on Days 5. $* * p<0.01$, significant difference to the control group; $\# p<0.05$, significant difference to the $\mathrm{G}+\mathrm{L}-\mathrm{P}$ treated group.

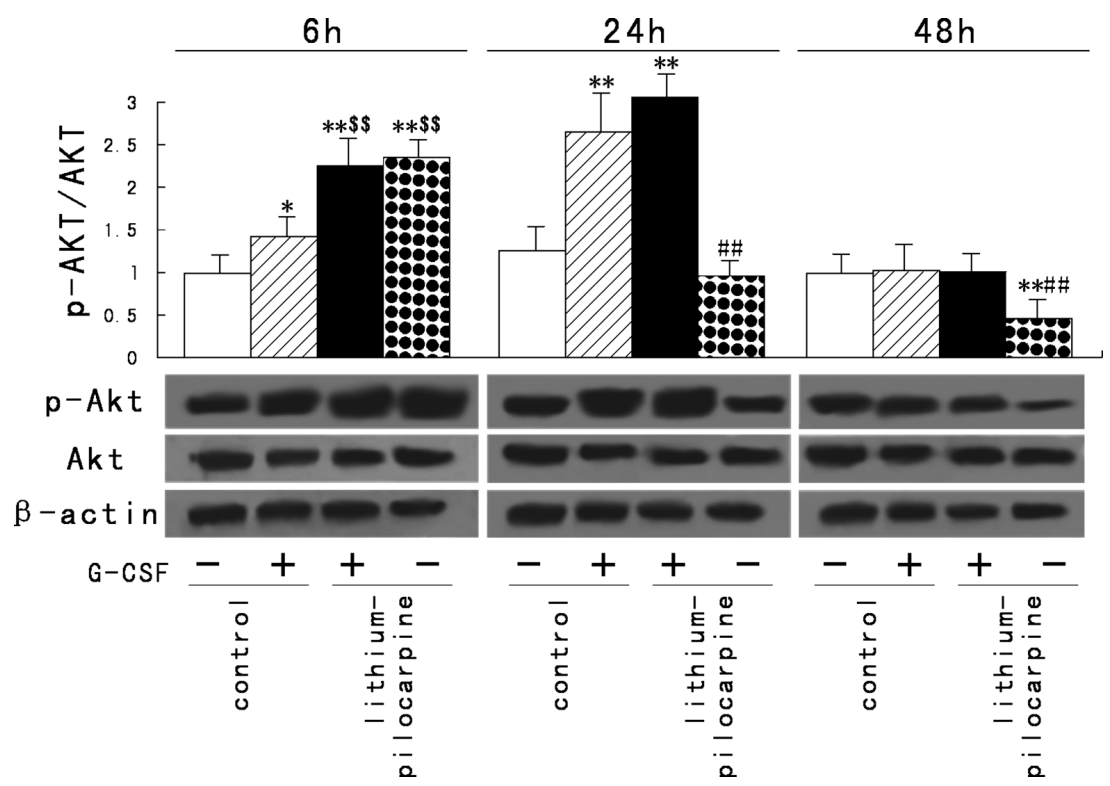

Fig. 7. Western Blot Analysis of Phosphorylated-AKT 1/2/3, Total AKT and $\beta$-Actin at the 6th, 24th and 48th Hour after the Treatment

$* * p<0.01, * p<0.05$, significant difference to the control group; \# $p<0.01$, significant difference to the G+L-P treated group; $\$ s<0.01$, significant difference to the G-CSF treated group. 
L-P treated groups were observed on both the third (Fig. 6I, $42.2 \pm 12.9$ vs. $2.5 \pm 1.9, p<0.01$ ) and the fifth days (Fig. 6J, $19.3 \pm 6.2$ vs. $1.8 \pm 1.4, p<0.01)$. G-CSF treatment antagonized the caspase- 3 cleavages induced by L-P injection on the third day $(26.4 \pm 9.2$ vs. $42.2 \pm 12.9, p<0.05)$ but had almost no effects to reduce the cleaved caspase- 3 positive cells on the fifth day $(17.7 \pm 7.1 v s .19 .3 \pm 6.2, p>0.05)$.

G-CSF Treatment Elicited AKT Phosphorylation in the Lithium-Pilocarpine-Induced SE Models Phosphatidylinositol 3-kinases (PI3K)-AKT signaling pathway is involved in cellular survival by inhibiting apoptotic processes. $^{26)}$ Our Western blot results demonstrated the phosphorylated-AKT 1/2/3 (pAKT) was significantly increased in G-CSF, L-P and G+L-P treated groups and obvious differences were present between G-CSF treated and the other two increased groups at the 6th hours after the treatment. At the 24th hour, pAKT was only significantly increased in G-CSF and $\mathrm{G}+\mathrm{L}-\mathrm{P}$ treated groups but decreased in the L-P treated group. In the 48th hours, no obvious changes of pAKT were observed within the G-CSF and G+L-P treated groups but dramatically decreased in the L-P treated group (Fig. 7).

\section{DISCUSSION}

To better understand the process of epileptogenesis and discover new therapeutic methods for epilepsy, several animal models had been developed to reproduce SE. Among them, the chemical convulsant pilocarpine was applied to produce acute seizures in a dose related manner, and that could culminate in an episode of SE. In this model, spontaneous seizures had been observed following a latent or maturational period that developed after an initial seizure episode. The model appeared to be highly isomorphic with the human temporal lobe epilepsy and was used to study the efficacy of antiepileptic drugs (AEDs). ${ }^{27)}$

Methods to detect apoptosis had been devised including TUNEL staining, in situ end labeling, DNA laddering analysis, membrane Annexin-V analysis or detection of apoptosis related proteins such as p53 and Fas. TUNEL staining was usually applied in tissues section analysis. ${ }^{28)}$ Lithium-pilocarpine treatment elicited TUNEL-positive neuron on hippocampal CA1 region had been described elsewhere. ${ }^{729)}$ In our study, we found the hippocampal neuronal apoptosis was increased after the L-P treatment as time went by because the elevated occurrences of TUNEL-positive neurons were observed from the 3 rd to the 5 th days. Then, the administered rhG-CSF decreased the numbers of TUNEL-positive neurons. Therefore, we inferred that G-CSF should exert antiapoptosis function. Herein, the way in which G-CSF acted should be explored in our system.

After seizure, both the intrinsic (mitochondrial) and extrinsic (death receptor) apoptotic pathways were activated. Two major families of genes, caspases and Bcl-2 family members, regulated apoptosis. Bcl-2 family was composed of proapoptotic (including Bax, BAD, Bak and Bok et al.), antiapoptotic (including $\mathrm{Bcl}-2$ proper, $\mathrm{Bcl}-\mathrm{xL}$ and $\mathrm{Bcl}-\mathrm{w}$ et al.) and $\mathrm{BH} 3$ domain-only (including Bid, Bad, Noxa and Puma et al.) members. They governed mitochondrial outer membrane permeabilization (MOMP). ${ }^{30,31)}$ and mainly participated in the intrinsic apoptotic pathway. The Bcl-2-associated $\mathrm{X}$ protein $(\mathrm{Bax})$ promoted apoptosis by competing with
Bcl-2 proper. Bax was a pro-apoptotic Bcl-2 protein and the majority of Bax was found in the cytosol of the normal mammalian cells. However, upon initiation of apoptotic signaling, Bax underwent a cascade of conformational changes from being inactive and cytosolic to an oligomeric, membrane-inserted state, then induced the opening of the mitochondrial voltage-dependent anion channel and led to activation of caspases. ${ }^{32)}$ The Bax expressions were increased in the SE models induced by lithium-pilocarpine or kainic acid. ${ }^{33,34)}$ One of the mechanisms involving the increased Bax expression was p53 induction following lithium-pilocarpine-mediated SE. ${ }^{35}$ In a renal ischaemia and reperfusion model, G-CSF treatment attenuated injury via activation of PI3/Akt signal pathway. ${ }^{36)}$ Activated PI3K/AKT could either increased Bcl-2 expression or directly phosphorylated and inactivated the proapoptotic function of Bax. ${ }^{37,38)}$ Therefore, we deduced that in our study, G-CSF increased Akt phosphorylation and the latter reduced Bax expression. Whether p53 played roles in the antiapoptotic effects elicited by G-CSF treatment still needed explored in our modle. The numerical alteration of Bax-positive neuron in our study reflected the expression of Bax induced by varied stimuli and it may be due to relative low sensitivity of the antibodies against Bax used here.

In contrast, Bcl-xL protein had anti-apoptotic functions by at least two distinct mechanisms; one was associated with heterodimerization and the other was with the ability to form a sustained ion channel. ${ }^{39)}$ In the kainic acid injection SE model, no Bcl-xL decline was detected. ${ }^{31)}$ The changes of Bcl-xL in this model were never mentioned before. Here, we found lithium-pilocarpine treatment decreased neuronal $\mathrm{Bcl}-$ $\mathrm{xL}$ expression sharply and increased the levels of Bax. The balance between these two molecules was disrupted. However, G-CSF increased the Bcl-xL level markedly and counteracted the decrease of Bcl-xL. Therefore, G-CSF rebuilt the balance between Bax and Bcl-xL, two major components in Bcl-2 family with opposite functions and hence decreased lithium-pilocarpine-induced neuronal apoptosis.

Caspases were a family of cysteine proteases, which played essential roles in extrinsic apoptotic pathways, necrosis and inflammation. Eleven caspases had so far been identified in humans and were divided into two kinds of apoptotic caspases: initiator (apical) caspases and effector (executioner) caspases. The initiator caspases (e.g. caspase-2, caspase-8, caspase-9 and caspase-10) cleaved inactive pro-forms of effector caspases, thereby activated them. The effector caspases (e.g. caspase-3, caspase-6, and caspase-7) in turn cleaved other protein substrates within the cell to trigger the apoptotic process. Caspase-3 was one of the key executioners of apoptosis, being responsible either totally or partially for the proteolytic cleavage of many key proteins. ${ }^{40)}$ In this study, it was found that lithium-pilocarpine treatment induced caspases-3 cleavage and G-CSF reduced the process. Meanwhile, in mammals, Bcl-2 and Bcl-XL block apoptosis by interfering with the activation of caspase-3. In the stroke models, G-CSF also decreased caspase-3 activation. Therefore, we deduced that G-CSF antagonized caspase-3 activation both via signaling pathways downstream of its receptor and activation of Bcl-2 family members.

Interaction between the specific growth factors with their membrane receptors would strengthen an important antiapoptosis system, PI3K and its direct downstream target 
AKT. Akt is phosphorylated (activated) in the human temporal lobe epilepsy (TLE) brain compared with control and its downstream molecules were observed as well. ${ }^{41)}$ Here, we found during the acute period after lithium-pilocarpine treatment ( $6 \mathrm{~h}$ after the treatment), AKT phosphorylation was increased (Fig. 7), which maybe due to the self-protection through self-secretion of certain proliferative cytokines. At the late phase of $48 \mathrm{~h}$, apoptosis evoked neuron loss and the amount of pAKT got decreased as well. We treated the rats with repeated G-CSF injections, which enhanced AKT phosphorylated and therefore counteracted the decrease of pAKT by lithium-pilocarpine treatment. Taken together, G-CSF protected the hippocampal neuron against apoptosis by enhancing AKT through G-CSFR and blocking both extrinsic and intrinsic apoptotic pathways in the lithium-pilocarpineinduced SE rats model.

In this assay, we found G-CSF administration helped the rats treated with lithium-pilocarpine keep the relatively normal body weight. The results of water maze showed G-CSF protected their learning ability and memory as well. The observations on apoptotic molecules correlated the protection for hippocampal neurons with improved cognitive ability. In clinic, repeated SE will cause significant cerebral damage and increased the risk of subsequent seizures, along with the hippocampal neuron loss and cognitive impairments. Epilepsy would lower the patients' living standard and strengthen their and the social burdens, especially in the developing countries. Therefore, except for the traditional antiepilepsy drugs, we also wanted to chase more effective supplements. Although it seemed promising for SE therapy with $\mathrm{G}-\mathrm{CSF}$ in rats model, whether it is effective in clinic still needs further studies.

\section{REFERENCES}

1) Scharfman H. E., Curr. Neurol. Neurosci. Rep., 7, 348-354 (2007).

2) Prasad A. N., Seshia S. S., Adv. Neurol., 97, 229-243 (2006).

3) Henshall D. C., Simon R. P., J. Cereb. Blood Flow Metab., 25, 15571572 (2005).

4) Huerta S., Goulet E. J., Huerta-Yepez S., Livingston E. H., J. Surg. Res., 139, 143-156 (2007).

5) Devinsky O., Epilepsia, 45, $34-40$ (2004).

6) Boon P., Raedt R., de Herdt V., Wyckhuys T., Vonck K., Neurotherapeutics, 6, 218-227 (2009).

7) Yang J., Huang Y., Yu X., Sun H., Li Y., Deng Y., Acta Neurobiol. Exp., 67, 141-148 (2007).

8) Jun Y., JiangTao X., YuanGui H., YongBin S., Jun Z., XiaoJun M., JianChun X., Heng X., XiaoXin Z., XinXiang X., Brain Res., 1282 $57-66$ (2009).

9) Solaroglu I., Cahill J., Tsubokawa T., Beskonakli E., Zhang J. H., Neurol. Res., 31, 167-172 (2009).

10) Matchett G. A., Calinisan J. B., Matchett G. C., Martin R. D., Zhang J. H., Brain Res., 1136, 200-207 (2007).

11) Kim N. K., Choi B. H., Huang X., Snyder B. J., Bukhari S., Kong T. H., Park H., Park H. C., Park S. R., Ha Y., Eur. J. Neurosci., 29, 891900 (2009).
12) Nishio Y., Koda M., Kamada T., Someya Y., Kadota R., Mannoji C., Miyashita T., Okada S., Okawa A., Moriya H., Yamazaki M., J. Neuropathol. Exp. Neurol., 66, 724-731 (2007).

13) Zavala F., Abad S., Ezine S., Taupin V., Masson A., Bach J. F., J. Immunol., 168, 2011-2019 (2002).

14) Schneider A., Kuhn H. G., Schäbitz W. R., Cell Cycle, 4, 1753-1757 (2005).

15) Matchett G. A., Calinisan J. B., Matchett G. C., Martin R. D., Zhang J. H., Brain Res., 1136, 200-207 (2007).

16) Zhao L. R., Navalitloha Y., Singhal S., Mehta J., Piao C. S., Guo W. P., Kessler J. A., Groothuis D. R., Exp. Neurol., 204, 569-573 (2007).

17) Lan X., Qu H., Yao W., Zhang C., Tohoku J. Exp. Med., 216, 117-126 (2008).

18) Toth Z. E., Leker R. R., Shahar T., Pastorino S., Szalayova I., Asemenew B., Key S., Parmelee A., Mayer B., Nemeth K., Bratincsák A., Mezey E., Blood, 111, 5544-5552 (2008).

19) Lu C. Z., Xiao B. G., Front. Biosci., 12, 2869-2875 (2007).

20) Nishio Y., Koda M., Kamada T., Someya Y., Kadota R., Mannoji C., Miyashita T., Okada S., Okawa A., Moriya H., Yamazaki M., J. Neuropathol. Exp. Neurol., 66, 724-731 (2007).

21) Meldrum B. S., J. Nutr., 130, 1007S-1015S (2000).

22) Racine R. J., Electroencephalogr. Clin. Neurophysiol., 32, 281-294 (1972).

23) Gerlai R., Behav. Brain Res., 125, 269-277 (2001).

24) Xie X., Wang C. Y., Cao Y. X., Wang W., Zhuang R., Chen L. H., Dang N. N., Fang L., Jin B. Q., World J. Gastroenterol., 11, 344-347 (2005).

25) Meller R., Clayton C., Torrey D. J., Schindler C. K., Lan J. Q., Cameron J. A., Chu X. P., Xiong Z. G., Simon R. P., Henshall D. C., Epilepsy Res., 70, 3-14 (2006).

26) Wang J., Wu A., Xu Y., Liu J., Qian X., Cancer Lett., 283, 193-202 (2009).

27) White H. S., Epilepsia, 38, S9-17 (1997).

28) Stadelmann C., Lassmann H. D., Cell Tissue Res., 301, 19-31 (2000).

29) Wang S., Wang S., Shan P., Song Z., Dai T., Wang R., Chi Z., Brain Res. Bull., 76, 90-96 (2008).

30) Murphy B., Dunleavy M., Shinoda S., Schindler C., Meller R., Bellver-Estelles C., Hatazaki S., Dicker P., Yamamoto A., Koegel I., Chu X., Wang W., Xiong Z., Prehn J., Simon R., Henshall D., Am. J. Pathol., 171, 1258-1268 (2007).

31) Chipuk J. E., Green D. R.,. Trends Cell Biol., 18, 157-164 (2008).

32) Adams J. M., Cory S., Curr. Opin. Immunol., 19, 488- 496 (2007).

33) Xu J., Wang S., Lin Y., Cao L., Wang R., Chi Z., Neurosci. Lett., 453, 58-61 (2009).

34) Mikati M. A., Zeinieh M., Habib R. A., El Hokayem J., Rahmeh A., El Sabban M., Usta J., Dbaibo G., Epilepsy Res., 81, 161-166 (2008).

35) Tan Z., Sankar R., Tu W., Shin D., Liu H., Wasterlain C. G., Schreiber S. S., Brain Res., 929, 129-138 (2002).

36) Li Y., Wu J., Shou Z., He Q., Zhang P., Han F., Li H., Chen J., Nephrology, 13, 508-516 (2008).

37) Asnaghi L., Calastretti A., Bevilacqua A., D’Agnano I., Gatti G., Canti G., Delia D., Capaccioli S., Nicolin A., Oncogene, 23, 5781-5791 (2004).

38) Xin M., Deng X., J. Biol. Chem., 280, 10781-10789 (2005).

39) Minn A. J., Kettlun C. S., Liang H., Kelekar A., Vander Heiden M. G., Chang B. S., Fesik S. W., Fill M., Thompson C. B., EMBO J., 18, 632-6243 (1999).

40) Mazumder S., Plesca D., Almasan A., Methods Mol. Biol., 414, 13$21(2008)$

41) Shinoda S., Schindler C. K., Meller R., So N. K., Araki T., Yamamoto A., Lan J. Q., Taki W., Simon R. P., Henshall D. C., J. Clin. Invest., 113, 1059-1068 (2004). 